Check for updates

Cite this: Lab Chip, 2021, 21, 987

DOI: 10.1039/d1lc90012a

\section{Musings on the future of scientific (physical but not socially distanced) conferences: testing the water with organizing the on-line MicroTAS2020}

Séverine Le Gac (iD *ab and Hang Lu (DD *c rsc.li/loc

As many people have experienced in the past year or so around the world, COVID19 has brought many changes in our lives. To scientists, this means, in addition to personal impact, changes around how science is done and communicated and in how ideas are exchanged. In many fields, including the lab-on-a-chip community, in-person conferences have been a major venue for show-casing trainees' work, exhibiting new technologies, exploring new frontiers, exchanging ideas, and building the community. With the COVID19 travel restrictions and with many labs being shut down temporarily, these inperson conferences were largely either pushed back at least another calendar year or turned virtual. We two had the opportunity to organize MicroTAS2020 (virtual) (Fig. 1), which was originally slated to take place in Palm Springs, CA, USA, and co-chaired by Prof. Amy Herr and Prof. Joel Voldman. The experience was largely positive, and it also prompted thoughts on opportunities that the new not-so-normal "normal" brought. Notably, going online for scientific meetings and/or adapting our travel habits was already on the agenda before the COVID-19 crisis, driven by the desire

\footnotetext{
${ }^{a}$ Applied Microfluidics for BioEngineering Research, MESA+ Institute for Nanotechnology \& TechMed Center, University of Twente, 7500 AE Enschede, The Netherlands.E-mail: s.legac@utwente.nl

${ }^{b}$ Faculty of Electrical Engineering, Mathematics and Computer Sciences, University of Twente, $7500 \mathrm{AE}$ Enschede, The Netherlands

${ }^{c}$ School of Chemical \& Biomolecular Engineering, Georgia Institute of Technology, 311 Ferst Dr. NW, Atlanta, GA 30332-0100, USA.

E-mail:hang.lu@gatech.edu
}

to cause less harm to our planet since scientists often belong to the $1 \%$ of frequent flyers and super-emitters. ${ }^{1}$ For these environmental reasons, in January 2020, POM, the Photonics Online Meetup, was the first scientific meeting organized fully online. ${ }^{2}$ The purpose of this article is to reflect on and share our adventure and view on new opportunities and best practices, and hopefully prompt the community to contribute to the conversations about how we move forward in the post-pandemic world.

\section{Origin of the virtual MicroTAS2020}

In response to travel restrictions and lab shutdowns, the CBMS (Chemical and Biological Microsystems Society) board of directors (the governing board for MicroTAS meetings) determined in April

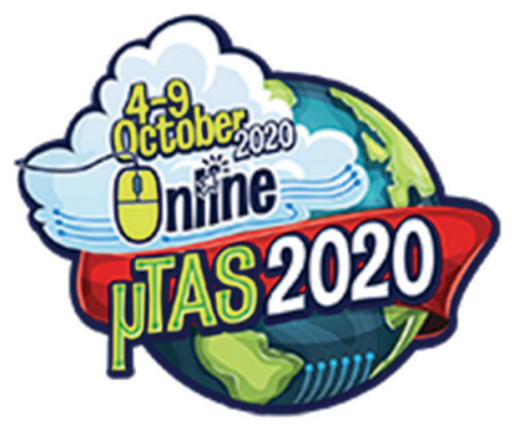

Fig. 1 Logo of the MicroTAS2020 conference, which took place online with live content scheduled on the 4-9th of October 2020. For more information on the conference content, please visit https:// microtas2020.org. The MicroTAS conferences are organized by the Chemical and Biological Microsystems Society (CBMS).
2020 that a virtual meeting was to take place at the originally designated time of the year since maintaining an in-person meeting in this pandemic context was too risky. The stated purpose was to make sure we continue to engage and strengthen our community in spite of these difficult lockdown times where some of our labs are entirely or partially closed. Many felt that junior researchers have the most to lose should these conferences not take place at all. Because this was the first virtual meeting for our community, and because there were multiple unknowns at the time, we envisioned a modified format (see below) that would still capture the spirit of MicroTAS: (1) to maintain the quality of the meeting; (2) to provide a forum for new/unpublished work; (3) to promote scientific engagement/exchanges in a wide variety of subject areas; (4) to promote exchanges among researchers of all career stages; and (5) to grow the community by engaging new scientific directions and new researchers. Upon consideration of the circumstances (e.g., number of potential abstracts, time zones, scientific content, and conferencing technologies available), we envisioned a one-week long meeting with 4-5 hours of synchronous and asynchronous components, as discussed in more detail below.

\section{Choice of the conference scientific content}

One of the main considerations for organizing meetings is the scope and consequently the format. What started 
as the organization of an event for the microfluidic community quickly turned into the organization of a "real" MicroTAS conference. Taking into account the pandemic context and the shorter time, choices had to be made on the actual scientific content of the conference. At a "traditional" MicroTAS, the way we have experienced it in the last $>15$ years, and like at many other conferences, the program comprises invited plenary talks, invited keynote presentations, selected oral presentations, selected posters and industrial stages. For MicroTAS2020, we decided to omit selected oral presentations from submitted abstracts, for the following reasons. Because the selection of talks is based on the quality of the submitted abstracts, the selection process this year could potentially disadvantage different labs due to the lockdown and lab closure policies. Since the program was restricted to about 5 hours a day, it was challenging to accommodate two sessions per day of selected talks and to cover all abstract categories, as normally happens. All other meeting features were kept, including six plenary speakers, eight keynote speakers organized as two sessions in parallel with four presentations each, and two one hour poster sessions per day. Industrial stages were also offered to benefactors in the same format as all invited scientific presentations. MicroTAS2020 also featured two award sessions: ${ }^{3}$ one for the two prestigious young-to-mid-career awards, the Young Innovator Award and the Pioneers in Miniaturization Lectureship Award; and one at the very end of the conference with all poster awards, the Art in Science Award, the Flash Presentation Award and the Best Paper award, the latter two being new to MicroTAS. Finally, short video clips were streamed at the beginning and end of every live session, in which scientists from the microfluidic community could provide a personal message for the MicroTAS conference (Fig. 2), companies could highlight products and information was provided on gaming activities.

\section{Technological choices}

One particularly challenging and unknown parameter when going online is the format of the conference and the technological platform that will house the entire conference. This puzzle took some time to be solved, since we strived for an affordable online option, while still offering a user-friendly and as integrated as possible interface. Notably, while online business has been blossoming in the last few months, driven by the urgency to have sophisticated online meeting environments, at the time when decisions needed to be made (late spring 2020), options were limited. After discussion with different colleagues and their positive experiences with the interface used for the annual meeting of the ASMS (American Society for Mass Spectrometry), we decided to adopt the same EventPilot platform for MicroTAS2020. Some of its attractive features are that it is an all-in-one platform with an intuitive interface, a detailed program and an online planner to customize one's conference schedule, embedded talks and posters, the opportunity for live sessions in Zoom (webinar) rooms, all exhibitors and benefactors, and the possibility to interact and chat with other attendees. Each presentation and/or live activity was allocated one page, with a link to a Zoom webinar, the corresponding proceedings, a link to the pre-recording for invited talks, and a link to the live recording after the event had taken place. Similarly, all the information related to each poster was collected on a single page, with the submitted conference proceeding, the poster in an A4 landscape format, the corresponding 1 min flash presentation, a link to a Zoom room active during the allocated poster session, as well as various options to engage with authors outside the allocated poster session. While at other online conferences posters may adopt a different format - e.g., 3-4 slides possibly with a voice over - this onepage overview format, as well as the 1 minute flash presentation for the authors of each paper to introduce their research, created an opportunity to provide content in a more dynamic manner. On the same platform, various chat channels, roughly corresponding to the abstract submission categories, were incorporated, as well as a job market and a list of all benefactors with some highlighted product information and possibly short video clips.

For live talks and Q\&As, we selected Slido as an additional technological platform for attendees to ask questions and session chairs to curate the questions. This platform allows live interactions, and in fact while talks were streamed, questions could already be asked. Also, Slido offers additional attractive features for voting on and ranking questions, and for archiving comments and questions, and it is easy to use on a phone, tablet or computer. Perhaps more importantly, using Slido, questions could be asked on a prerecorded presentation before the Zoom webinar opened and they remained there after the session was terminated. Slido also bypasses the Zoom webinar (which, in its embedded form in the EventPilot platform, does not permit question submission).

\section{An online meeting with no digital professionals}

No digital professionals were hired for any technological aspects before and during the conference. Instead, a dedicated committee formed by colleagues from the microfluidics community - those with an affinity for technology - was appointed. This committee was critical for the success of the meeting. This committee's primary function was to anticipate possible problems linked to the online format (handling of the digital environment, preparation of the digital posters and 1 min video, pre-recording of all oral presentations, etc.) and to in turn propose timely solutions. Eventually, the same virtual technical committee (VTC) ran all Zoom webinars and live events during the conference (Fig. 3), with the assistance of a group of volunteering students/young researchers. Each activity in the Zoom 

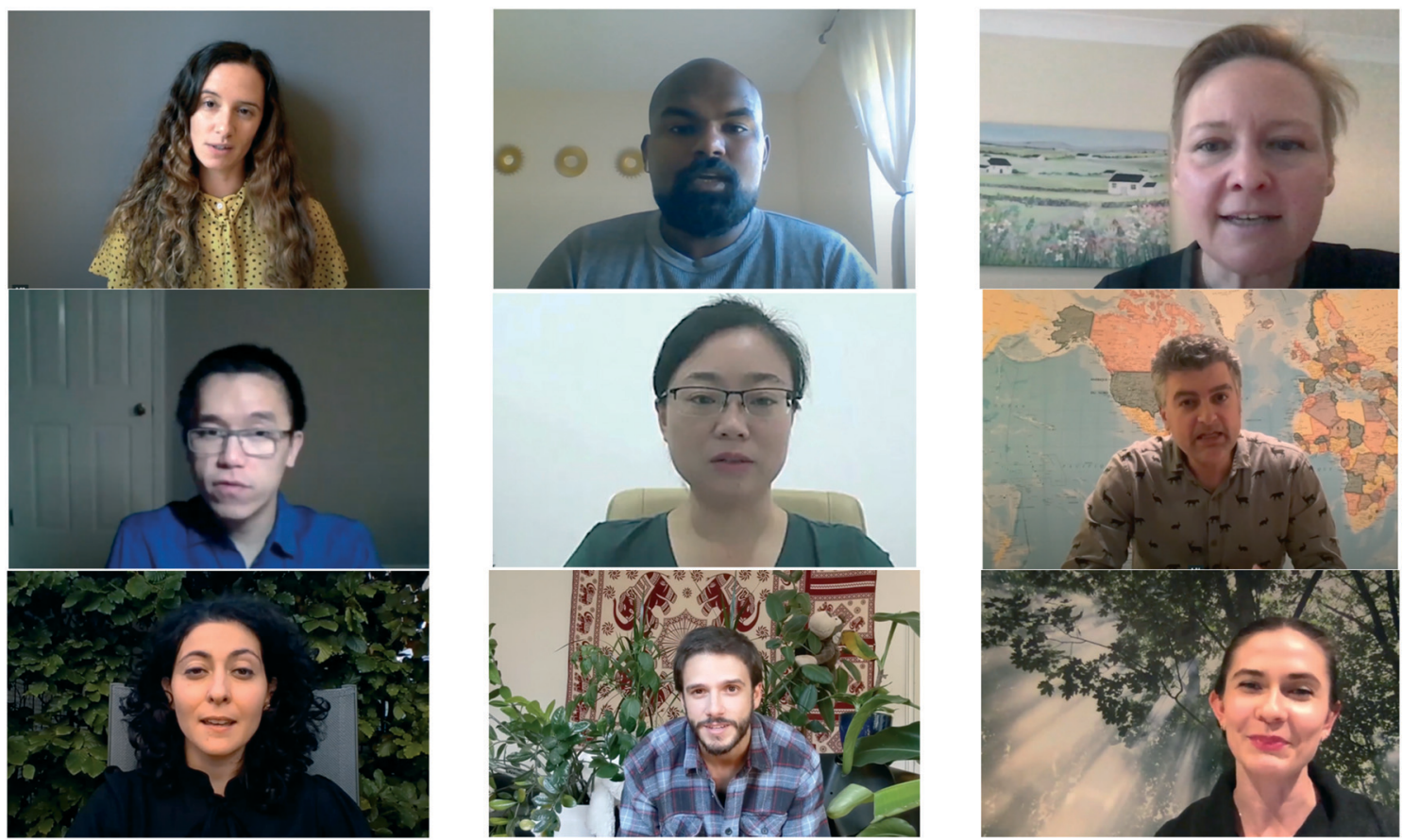

Fig. 2 Snapshots of some video contributions from members of the microfluidic community highlighting what the MicroTAS conference means to them, the importance of having a MicroTAS conference in this COVID-19 situation and the foreseen advantages of an online conference. Picture featuring, from left to right in each row: Dr Katherine Elvira, Dr Michael Issac, Prof. Nicole Pamme, Dr Gongchen Sun, Prof. Lingling Shui, Dr Thomas Gervais, Dr Burcu Gumuscu, Nassim Rousset and Dr Samantha Grist.

rooms (except for individual posters) was run by two people, one VTC member and one Zoom-helper, in two very different geographical locations to avoid potential internet glitches, and two senior VTC members were always available to jump in in case of unforeseen problems. As a result, this choice to only rely on non-professional volunteers carried potential risks, but no major incident happened, and small technical glitches were readily resolved by the team, without any consequence for the overall progress/flow of the conference.
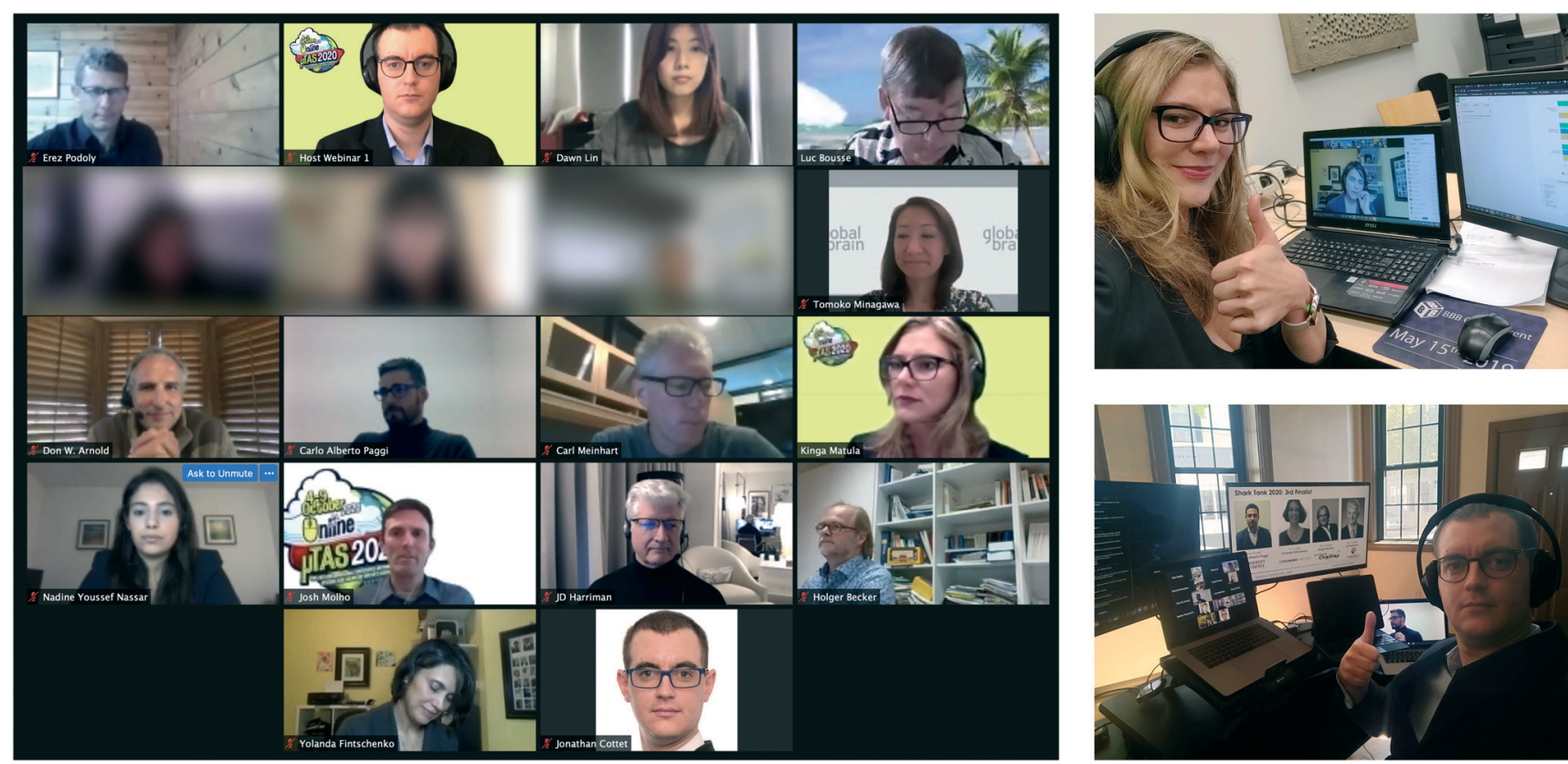

Fig. 3 One of the most challenging moments from a technical point of view, the shark tank, with 18 people online simultaneously, with the two VTC members (Dr Kinga Matula and Dr Jonathan Cottet) in charge of this live moment. 


\section{Asynchronous and synchronous content}

The conference was limited to about five hours a day, at the "best" time to accommodate the time differences between all attendees worldwide (i.e., 8 am to $1 \mathrm{pm}$ EST), and following the best practices for international online conferences. Still, this time window is far from being ideal for all attendees, especially those from Asia and Oceania, which together represent a significant share of the MicroTAS audience. Therefore, almost all conference content was also available in an asynchronous manner on the platform, for attendees to enjoy talks, posters and live events at the time of their preference. Specifically, all posters and their corresponding flash presentations were already offered online one week before the conference, together with the pre-recorded invited talks (keynote and plenary, industrial stages, etc.). The rationale behind this was that attendees could watch presentations before the time at which they were scheduled in the conference program, and ask questions via Slido with the option to follow the postpresentation live Q\&A session at a later moment. Therefore, these live Q\&A sessions, the speakers' corners and all live events (shark tank, panel discussions, closing ceremony, etc.) in the program were recorded and made available online on the platform for the rest of the conference, and one week after. While we do not have a quantitative assessment, anecdotal evidence suggests that the asynchronous content was helpful to attendees; many also commented that it was a bonus that no one needed to miss some keynote talks because they were pre-recorded (in contrast with in-person meetings where one could only attend one keynote talk among three parallel sessions). For this meeting, plenary and keynote talks were also planned at different times on different days in the program so as not to disadvantage one region of the world and to take into account the time zones of the speakers. This combination of synchronous and asynchronous content, as summarized in Table 1, allows maintaining the feeling of having a conference at given times with a coherent daily program, while offering more flexibility to attendees in their planning as well as the opportunity to attend all content, which is not possible at in-person conferences for presentations taking place at the same time. Notably, this flexibility in accessing the scientific content is often mentioned as a key advantage of online conferences, as it allows attendees to avoid missing talks, for instance, or to watch them at a time of their choice.

\section{How to stimulate interactions?}

Interactions form the core of scientific conferences and have become one of the raisons d'être for many scientists, now that all scientific information becomes quickly available online. Interactions at scientific conferences come in different flavors: as scientific exchanges after talks and during poster sessions or between sessions, as new collaborations discussed in the corridors, as professional networking, or as more social elements and "fun". All these interactions take place in an $a d$ hoc and spontaneous fashion between attendees when they accidentally bump into each other in the conference physical space. We therefore appointed a dedicated social committee to reflect on these social elements and to organize activities to foster interactions beyond pure science. Social events are actually an entire part of in-person conferences, and MicroTAS typically features organized evening events (women's night, students' get together and a conference dinner) and coffee breaks, as well as all the other spontaneous interactions between attendees.

Brainstorming went in different directions to eventually select the following activities (see Box 1 for more information), which were all meant to engage attendees in non-purely scientific aspects: a shark tank which has already been featured twice at MicroTAS in 2017 and 2018, and was thought to lend itself well to an online format; several panel discussions, which are arguably one of the main challenges of online conferences; scientific exchanges which are driven by the conference content, which can "easily" be maintained; other interactions, which are rather new for the MicroTAS conference; and several gaming activities, which were offered in a synchronous or asynchronous manner.

Also, as a means to foster interactions between attendees, the live Q\&A sessions after the talks were longer, and continued as a speakers' corner, which was further stimulated by the two chairs of the session. Each poster's presenter was provided with a Zoom room for the one-hour duration of their poster session; attendees could visit the poster presenter and interact with them in this room. We considered this conference activity to be central to the mission, and without it, scientific exchanges around posters could be drastically limited.

\section{Benefactors and sponsorship}

The industrial exhibition is an important feature of certain scientific conferences, as for instance MicroTAS. This exhibition serves different purposes: for companies to release and highlight new products; for researchers to discover new commercial solutions and purchase new equipment for their labs; for companies to identify new needs of researchers; and for companies and researchers to simply interact around the displayed set-up and equipment, and to possibly start new public-private partnerships. The exhibition is usually combined with the poster exhibition and the place for the coffee breaks to maximize interactions, a set-up that cannot easily be reproduced online, without using virtual reality. Sponsorship and exhibition options were adapted to this online format, to ensure proper visibility of the benefactors and offer different means to foster interactions with attendees. First, all benefactors were assigned one page on the conference website and on the digital platform, on which they could 


\section{Box 1 Social activities offered at MicroTAS2020.}

Three different types of social activities were offered at MicroTAS2020:
1. Panel discussions
2. A shark tank
3. Gaming activities

Panel discussions were moderated by two co-chairs and featured three to six panelists. Selected topics were: Woman in Academia, Ethics in Science, Advice for Early Careers in Academia, Women in Industry, Parenting in Science and Work-Life Balance and Students and Postdocs Studying Abroad. After a brief round of introductions where each panelist would introduce himself/herself, questions raised by the audience were asked to one or multiple panelists, and the debate was facilitated by the two chairs. For each panel discussion, panelists were selected to maintain geographical and gender (when applicable) diversity.

The shark tank featured three candidates selected by a panel of 11 judges based on their pre-conference application. Each candidate first pitched their product and business idea in a pre-recorded movie, before they were exposed to live questions from 3-4 judges. Business-related prizes were offered to the winners such as in-kind provisional patent filings and personal advice, as well as two prizes of \$2000 and \$1000.

Gaming activities came in three different flavors. First, daily live quizzes were organized by industrial partners on their products. Second, daily asynchronous quizzes on the program of the day were posed online until midnight EST and rated every day. Finally, a live game show "Who wants to be a millionthaire?" took place on the last day of the conference before the award ceremony; winners of the daily online quizzes competed in this final game together with 2 other people selected on the spot.
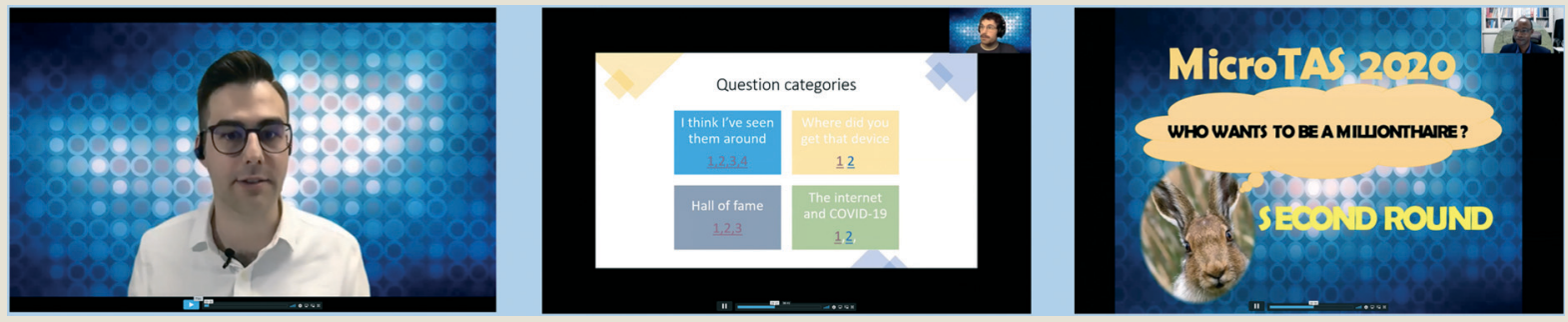

Figure Box 1: Who wants to be a millionthaire? Live game show occurring live at the end of the conference. Extracted pictures showing the three masters of ceremony - from left to right, Dr Darius Rackys, Dr James Perry and Dr Kennedy Okeyo.

highlight selected products, with a link to their website and email address, and a list of activities in which they would participate at the conference (e.g. industrial stage, poster, etc.). Online industrial stages were organized as any other oral presentation, in a prerecorded format, followed by a live Q\&A session moderated by two chairs. One clear advantage of the online format is, in our opinion, the possibility of including live demos, which are often difficult to set up at a conference. This year, to highlight some new developments or products, benefactors could present scientific posters, which were fully integrated with other posters selected based on abstract submission, and/or provide a short video-clip that was streamed at the beginning and end of the live sessions. Finally, several benefactors organized daily quizzes with questions on their products to encourage attendees to interact with companies. Yet, feedback indicated that interactions with companies were significantly reduced this year, which shows that translating the industrial exhibition online should remain a point of attention for future online scientific meetings, for instance using platforms offering virtual reality (VR) options.

\section{In hindsight - a well- organized team and structure}

As for any other MicroTAS and other conferences, this online event was organized by the chairs, us, with the assistance of the professional conference organization Preferred Meeting Management, Inc. and several committees in charge of specific aspects. Considering the short time frame ( $<5$ months) for the overall conference organization, frequent and regular meetings were held between the organizers, within the different subcommittees and between the chairs and the sub-committees. A shared working environment in the form of a Google Doc was essential for preparing all meeting agendas, keeping a living list of action points, and for joint writing activities, together with a joint repository for sharing all important files. A Slack channel was also set up by the VTC, which became particularly handy during the conference. Having such a well-organized structure was key, since all committees were assembled with representatives from all over the world, taking advantage of this online format to respect geographical diversity as well as gender balance, and not have representatives from one specific 
Table 1 Summary of the scientific content offered at MicroTAS2020, specifying its synchronous or asynchronous nature (live, pre-recorded, streaming, recording available online, etc.)

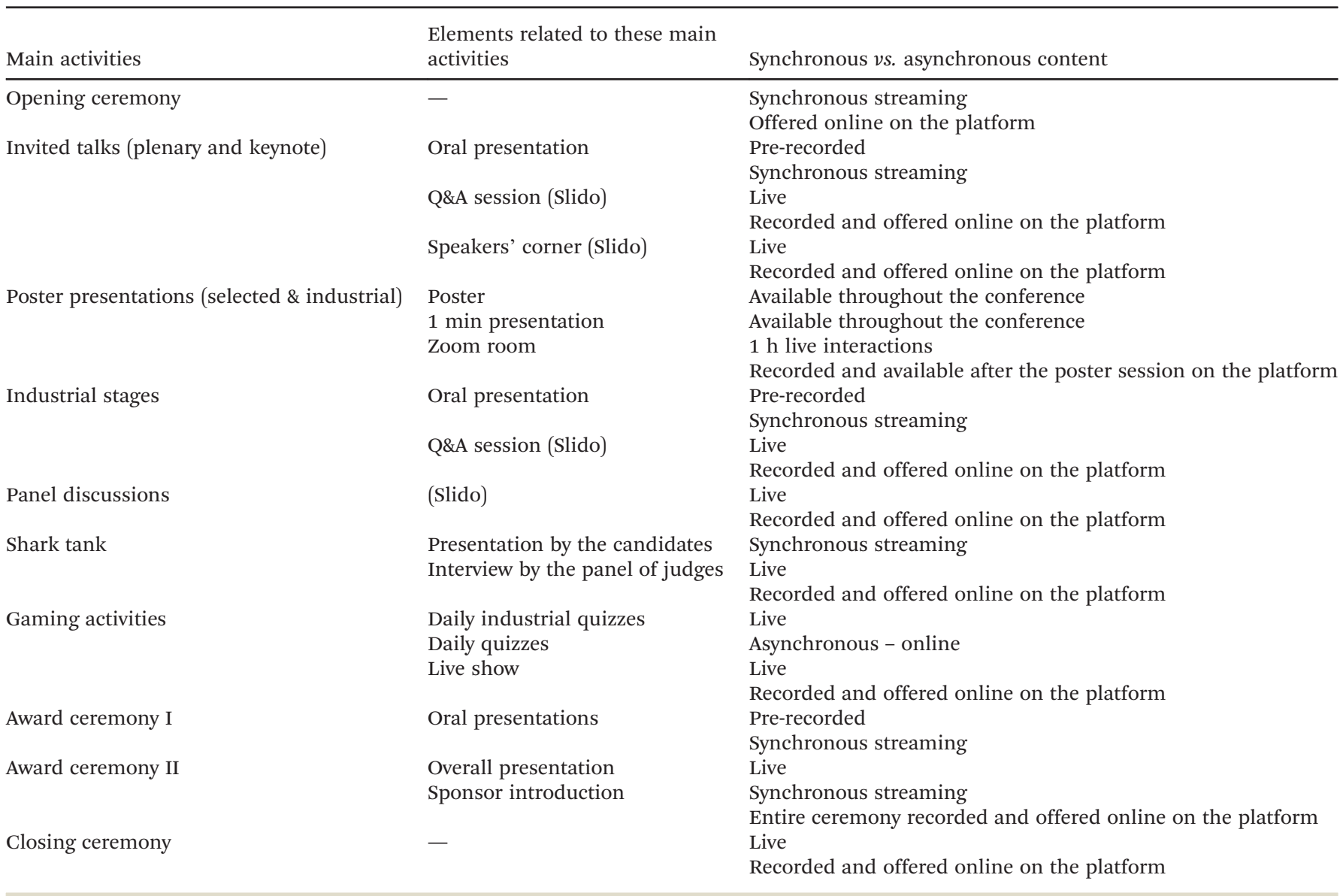

area, as would typically happen for inperson meetings. Yet, this geographical spread and time zone differences had to be compensated for by another organizational approach.

As with physical in-person meetings, professional conduct was expected of all attendees and organizers. We updated the CBMS code of conduct for all MicroTAS meetings to include online behavior (e.g. prohibition of cyber stalking); all attendees were reminded of the policies prior to and at the meeting. As a result, no incidents were reported.

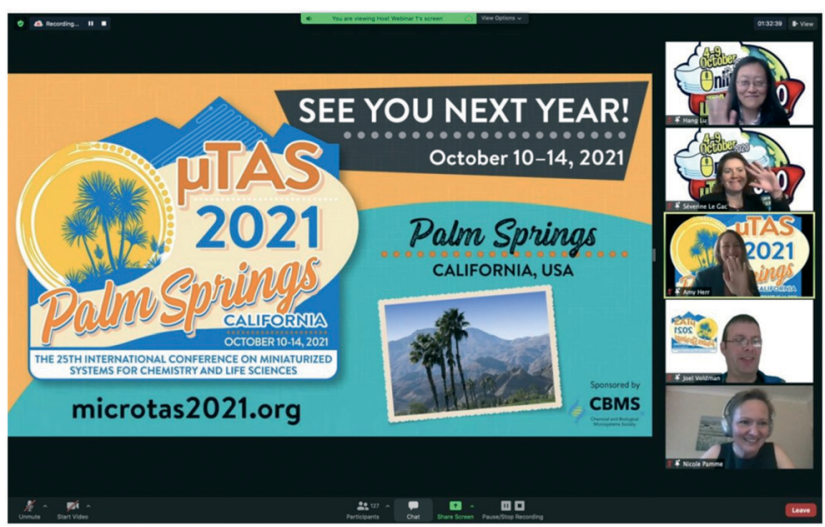

Fig. 4 Rounding up the week-long online MicroTAS2020 and looking to the future MicroTAS2021, picture featuring from top to bottom: Prof. Hang Lu, Prof. Séverine Le Gac, Prof. Amy Herr, Prof. Joel Voldman, and Prof. Nicole Pamme.

\section{Thinking to the future}

Feedback from the community on MicroTAS2020 has been largely positive and many colleagues and friends congratulated us on this achievement. Importantly, several new elements of the virtual meeting were deemed effective. Many thought that the use of 1 min flash presentations for the posters was a very effective way of screening the contents of the posters. The $1 \mathrm{~min}$ presentation was not onerous to review but delivered the best information content and provided effective advertisement of the work. Some authors seemed to have enjoyed the process of preparing creative content using these videos. A possible addition mentioned as feedback would be to create a poster gallery to emulate the feeling of a poster hall, which would also avoid multiple clicking to finally access the content of all posters in one particular session. The panel discussions were also 
positively welcomed by attendees, and such activities, which are considered as good practice and make up entire parts of other meetings such as the Gordon conferences, can easily be maintained for in-person or hybrid meetings, to reflect on essential aspects associated with science and researchers' lives. Many people, especially those in different time zones (or those with conflicting duties, e.g., teaching or other meetings), also enjoyed the ability to view content asynchronously. Talks being available prior to and after the scheduled time on the program made the content more accessible; this reduced the stress of having to choose between parallel sessions as at in-person meetings. Being able to view the recorded Q\&A sessions was also useful for some. Technologies such as Slido for asking, queuing, and curating questions were also a success. We feel that using this technological platform also lowered the psychological barrier of asking questions, especially for younger researchers. Many session chairs also liked the ability to organize the Q\&A sessions. Some of these elements can be easily translated to inperson or hybrid meeting formats with small tweaks and are therefore worth evaluating for future meetings.

Since MicroTAS2020 was organized at short notice ( $<5$ months) with no precedents for both the organization and the technologies needed, we hope some "hiccups" are understandable. It was certainly an experiment that we and the CBMS were willing to perform, and lessons learned can help improve future meetings. One of the main concerns was the ability to socialize and network. While Q\&A sessions, panel discussions, and Zoom rooms provided opportunities for interactions, those online interactions may not have been as organic and natural as in-person interactions would have been. At the time when most decisions on platforms and format were made (MayJune), some technologies that have now been tested to facilitate social interactions (e.g. spatial.chat, gather.town) had not been widely adopted and did not come up on our radar screens. These newer platforms using virtual reality (VR) can perhaps better meet the social requirements of large meetings like
MicroTAS where one can "walk up" to a presenter, or visit a virtual booth at the exhibition, or hang around a virtual coffee table or bar to have conversations with a flexible number of people. Finally, we think it is important for organizers to think about how to use elements of the meeting to create a full conference feeling, rather than disjointed components of a meeting.

Regardless of what the postpandemic world looks like, it seems to us that there are some opportunities to take advantage of for in-person or hybrid meetings, as well as newly emergent challenges. First, there are elements such as the $1 \mathrm{~min}$ video introductions to posters, asynchronous talks, use of a digital platform to ask questions and asynchronous feedback to presenters, which can be incorporated into the in-person or hybrid format, either as supplements or replacements. Second, some materials from the meeting are highly valuable as educational materials, e.g. as supplements to university teaching content for graduate and advanced undergraduate material, or as training materials for use in research or commercial settings. It is worth noting that the meeting promotes frontiers of research, and therefore at the time of abstract submission, research results are unpublished. Maintaining this tradition is necessary to ensure the meeting's high quality, but care must be taken to consider unpublished content that is now more easily accessible online. It is worthy for the community to explore adapting to norms such as publishing in archives (e.g. bioRxiv, medRxiv, and arXiv). Third, many have recognized that the pandemic lockdown and travel restrictions brought access opportunities: those who could not travel easily for financial or logistical reasons now have access, including researchers from the developing world, researchers with child or elderly care duties, or researchers who normally need to go through the lengthy process of obtaining a visa; because of the virtual format, the logistics are simpler, and costs are down, so more trainees can afford to attend (we do recognize that some countries have not allowed the flexibility of reassigning "travel" funds to attend conferences online, but we expect this will change soon). The reduced travel also has the added benefit of reducing the carbon footprint of individuals, which many attendees feel is in line with their personal choice (of, e.g. being environmentally conscious) and is increasingly in line with the policies of many universities and government agencies. Fourth, we worked hard, as the in-person meeting organizers in the past have done, to put together a diverse meeting, in terms of scientific area, geographical location, gender, race, ethnicity, sexual orientation, career stage, socioeconomic background, culture, etc. Whether the virtual format did better or worse compared to in-person meetings in terms of diversity and inclusion remains to be teased out as data are not abundant yet, but we note that these are issues that need continued attention.

The pandemic's end seems to be in sight with the development of the vaccines. While 2020 has been a challenging year for many, we hope that we learned as a community from the collective experiences. We urge future meeting organizers and societies sponsoring scientific meetings to take this opportunity to reflect and innovate promoting elements that have been tried and successful in bringing the scientific community together and promoting dialogue and addressing deficiencies in areas of concern. We look forward to seeing our community together at many more meetings to come (Fig. 4), inperson, virtual, or hybrid.

\section{References}

1 Guardian article on Covid and the environment, Available from: https:// www.theguardian.com/business/2020/ nov/17/people-cause-global-aviationemissions-study-covid-19.

2 O. Reshef, et al., How to organize an online conference, Nat. Rev. Mater., 2020, 5(4), 253-256.

3 MicroTAS2020 awards website, Available from: https://microtas2020. org/awards/awards_overview.html. 